\title{
Modeling electronic quantum transport with machine learning
}

\author{
Alejandro Lopez-Bezanilla ${ }^{1, *}$ and O. Anatole von Lilienfeld ${ }^{2,3, \dagger}$ \\ ${ }^{1}$ Materials Science Division, Argonne National Laboratory, 9700 S. Cass Avenue, Lemont, Illinois 60439, USA \\ ${ }^{2}$ Argonne Leadership Computing Facility, Argonne National Laboratory, 9700 S. Cass Avenue, Lemont, Illinois 60439, USA \\ ${ }^{3}$ Institute of Physical Chemistry, Department of Chemistry, University of Basel, Klingelbergstrasse 80, CH-4056 Basel, Switzerland
}

(Received 15 October 2013; revised manuscript received 3 April 2014; published 11 June 2014)

\begin{abstract}
We present a machine learning approach to solve electronic quantum transport equations of one-dimensional nanostructures. The transmission coefficients of disordered systems were computed to provide training and test data sets to the machine. The system's representation encodes energetic as well as geometrical information to characterize similarities between disordered configurations, while the Euclidean norm is used as a measure of similarity. Errors for out-of-sample predictions systematically decrease with training set size, enabling the accurate and fast prediction of new transmission coefficients. The remarkable performance of our model to capture the complexity of interference phenomena lends further support to its viability in dealing with transport problems of undulatory nature.
\end{abstract}

DOI: 10.1103/PhysRevB.89.235411

PACS number(s): 73.63.-b, 02.60.Lj, 89.20.Ff

\section{INTRODUCTION}

Substantial advances in computational science capabilities have opened new research frontiers, greatly expanding the impact of the material sciences community's work. The unrelenting drive towards the use of atomistic simulation for the routine generation of large data sets is obtaining great interest [1]. Large-scale efforts such as the United States Materials Genome Initiative [2] are aiming for the discovery and development of new compounds thanks to increasingly faster and cheaper computational resources [3]. In parallel, the development of data mining techniques for intelligent interrogation of large databases are succeeding in recognizing meaningful patterns in structured data [4,5]. It still remains an open question, however, as to how to integrate into explicit structure-property relationships the knowledge hidden, yet implicitly present, in the data. Machine learning (ML), the ability of computer algorithms to comprehend data and infer new results for new situations, is gaining importance as a tool of choice to analyze the growing and complex data generated in many scientific and engineering contexts [6,7]. By appropriately estimating pairwise distances in a data set, supervised learning techniques directly allow for the resolution of computationally expensive sets of equations by making sense of accumulated knowledge. Within atomistic simulation, ML already demonstrated its usefulness in predicting outcomes from known patterns and inferring new knowledge. Examples include chemical binding [8], electronic levels [9], and one-dimensional orbital free density functionals [10].

In this paper we use ML to develop a new and alternative ansatz for modeling transmission coefficients of disordered one-dimensional (1D) device channels. This resulting technique allows us to estimate the conductivity of a large data set of model device channels accurately, with very moderate computational effort. The model introduced predicts a real-valued function (electron transmission) for independent values (electron energy) based on training for examples that

\footnotetext{
*alejandrolb@gmail.com

†anatole.vonlilienfeld@unibas.ch
}

were previously constructed by randomly selecting a set of disordered systems. We validate the expected computed function with a second set of reference results. The ML model is able to capture the complex behavior of reflective electron waves canceling each other as a consequence of destructive interference upon multiple reflections between channel impurities. As such, this statistical model retains the underlying quantum features of the training data, and projects them into the validating set with high accuracy.

\section{METHOD}

We simulate electron transport for a conducting channel model, which is assumed to be sufficiently transferable. The system consists of an infinite-long hexagonal network of single-orbital atoms (simple model for a graphene nanoribbon) divided in three regions [see Fig. 1(a)]. A central region (channel) exhibiting geometrical or compositional disorder is coupled to the left and right to two semi-infinite and multitransverse mode ballistic leads. All backscattering phenomena occur in the channel, which matches the leads with reflectionless contacts.

A standard first-neighbor tight-binding Hamiltonian parametrizes the energetic description of the disordered system,

$$
H=\sum_{i} \epsilon_{i}|i\rangle\left\langle i\left|+\sum_{i, j} \gamma_{i j}\right| i\right\rangle\langle j|,
$$

where $\epsilon_{i}$ is the on-site energy of site $i$, and $\gamma_{i j}$ is the hopping element to a nearest-neighbor site $j$ in the lattice. In order to analyze the two-terminal transport through the conducting system, we use the Landauer-Büttiker (LB) approach [11-13] that provides a conceptually simple framework to describe the physics of electron coherent transport at the nanoscale. In a two-probe system, the conductance is quantized in $G_{0}=e^{2} / h$, the quantum unit of conductance, and reads:

$$
G(E)=G_{0} T(E)=G_{0} \sum_{n=1}^{N} T_{n}(E),
$$


(a)

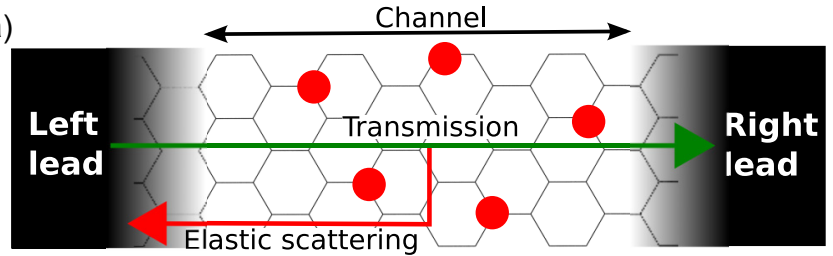

(b)

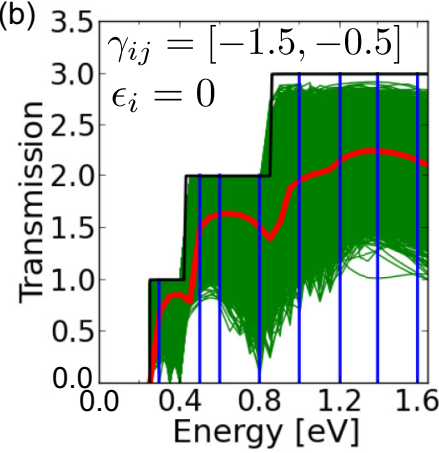

(c)

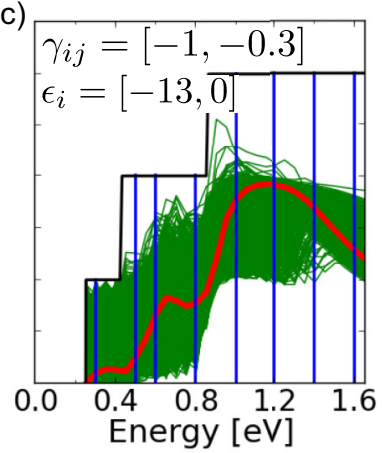

FIG. 1. (Color online) (a) schematic diagram of the modeled system. A hexagonal network of atoms divided in three parts: left and right leads, and a central region containing scattering centers. (b) and (c) show the transmissions of defectless channels (black lines), and of 8000 defective configurations for five spatially fixed scattering centers with the parameters in inset (green lines). Red lines indicate the average transmissions, and vertical blue lines the energies at which the machine has been trained for results in Fig. 2 and Fig. 3.

where the transmission coefficients $T(E)$ can be expressed as a sum over all the $N$ transmitting modes at energy $E$, and give the probability of an electron to be transmitted from one electrode to the opposite when it quantum mechanically interferes with the channel impurities. We evaluate the retarded (advanced) Green's functions of the system within the standard Green's function formalism,

$$
\mathcal{G}^{ \pm}(E)=\left\{E I-H-\Sigma_{L}^{ \pm}(E)-\Sigma_{R}^{ \pm}(E)\right\}^{-1},
$$

where $\Sigma_{L(R)}^{ \pm}(E)$ are the self-energies, which describe the coupling of the channel to the left $(-)$ and right $(+)$ electrodes. These quantities are related to the transmission factor by the relation [14],

$$
T(E)=\operatorname{tr}\left\{\Gamma_{L}(E) \mathcal{G}^{+}(E) \Gamma_{R}(E) \mathcal{G}^{-}(E)\right\}
$$

with $\Gamma_{L(R)}(E)=i\left\{\Sigma_{L(R)}^{+}(E)-\Sigma_{L(R)}^{-}(E)\right\}$. We have implemented and applied this framework for the generation of the various test and training sets outlined below.

An efficient supervised learning scheme relies upon a proper definition of a measure of similarity between systems. Probably the most crucial step consists of finding a suitable representation, also known as a descriptor [15], which should fulfill certain requirements such as symmetry invariance, uniqueness, or differentiability. Our electron transport model relies on the following square matrix $\mathbf{M}$ as a descriptor,

$$
M_{i j}= \begin{cases}\epsilon_{i} & \forall i=j, \\ \gamma_{i j} & \forall i \text { adjacent } j, \\ \sqrt{d_{i j}} & \text { otherwise, }\end{cases}
$$

where $d_{i j}=\left|\mathbf{R}_{i}-\mathbf{R}_{j}\right|$ is the distance between two sites. The dimensionality of $\mathbf{M}$ is the number of atoms in the channel.
Invariance with respect to site indexing is enforced by sorting the atom site indices according to the norm of the rows of $\mathbf{M}$. Note that $\mathbf{M}$ encodes the system's identity in terms of energetic descriptions and geometrical configurations, the same information that also defines the Hamiltonian entering the Green's function formalism.

For the ML model we rely on the standard Laplacian kernel model that has already been used in the context of a wide range of applications [16]. This ML model estimates the transmission $T$ at energy $E$ for a system with descriptor $\mathbf{M}_{J}$ as a sum of weighted exponential functions,

$$
T^{\mathrm{est}}\left(E, \mathbf{M}_{J}\right)=\sum_{I=1}^{N_{t}} \alpha_{I}(E) e^{-\frac{D_{I J}}{\sigma}},
$$

where $N_{t}$ is the number of samples in the training set, and where $D_{I J}=\left|\mathbf{M}_{I}-\mathbf{M}_{J}\right|$, i.e., the Euclidean norm between two channels $I$ and $J$. The regression coefficients $\left\{\alpha_{I}(E)\right\}$, and length scale $\sigma(E)$, are obtained at discrete values of $E$ through kernel ridge regression. The direct solution for the coefficient vector $\boldsymbol{\alpha}$ can be obtained through inversion of the training set's kernel matrix

$$
\boldsymbol{\alpha}(E)=[\mathbf{K}-\lambda \mathbf{I}]^{-1} \mathbf{T}^{\mathrm{ref}}(E),
$$

where $\mathbf{K}$ is the kernel matrix with elements $K_{I J}=e^{-D_{I J} / \sigma}$, and where $\mathbf{T}^{\mathrm{ref}}(E)$ is the vector of the training set's reference transmission coefficients at $E$. Since the employed reference data is noise free, the regularization parameter $\lambda$, ordinarily used to account for the noise in experimental data [16], has been set to zero. The characteristic length yielding optimal predictive performance of the model converged to $\sigma \sim 1000$. We have found little sensitivity with respect to variation of $\sigma$, and have therefore fixed it to $\sigma=1000$ for all predictions made in this study. It is worth pointing out that in Eq. (7) the kernel matrix inversion is independent of $E$. Since $\sigma$ is kept fixed this implies that for a given training set of channel configurations, only a single kernel matrix inversion is necessary to map out the entire set of corresponding channel weights $\left\{\alpha_{I}(E)\right\}$ as a function of $E$.

We estimate the performance of resulting models for predicting transmissions of out-of-sample systems by means of the mean absolute error (MAE) as measured with respect to corresponding reference transmissions

$$
\operatorname{MAE}(E)=\frac{1}{N_{t}} \sum_{i=1}^{N_{t}}\left|T_{i}^{\mathrm{est}}(E)-T_{i}^{\mathrm{ref}}(E)\right|,
$$

where $T_{i}^{\text {est }}(E)$ and $T_{i}^{\text {ref }}(E)$ are the respective predicted [Eq. (6)] and reference [Eq. (4)] transmissions at a given energy $E$.

\section{RESULTS AND DISCUSSION}

Transport calculations adopting the aforementioned Green's function formulation have been used to generate several training and testing data sets. For all experiments, impurities have randomly been scattered along a channel formed by repeating four times an armchair graphene nanoribbon unit cell with seven single-orbital atom dimers across the ribbon width. 

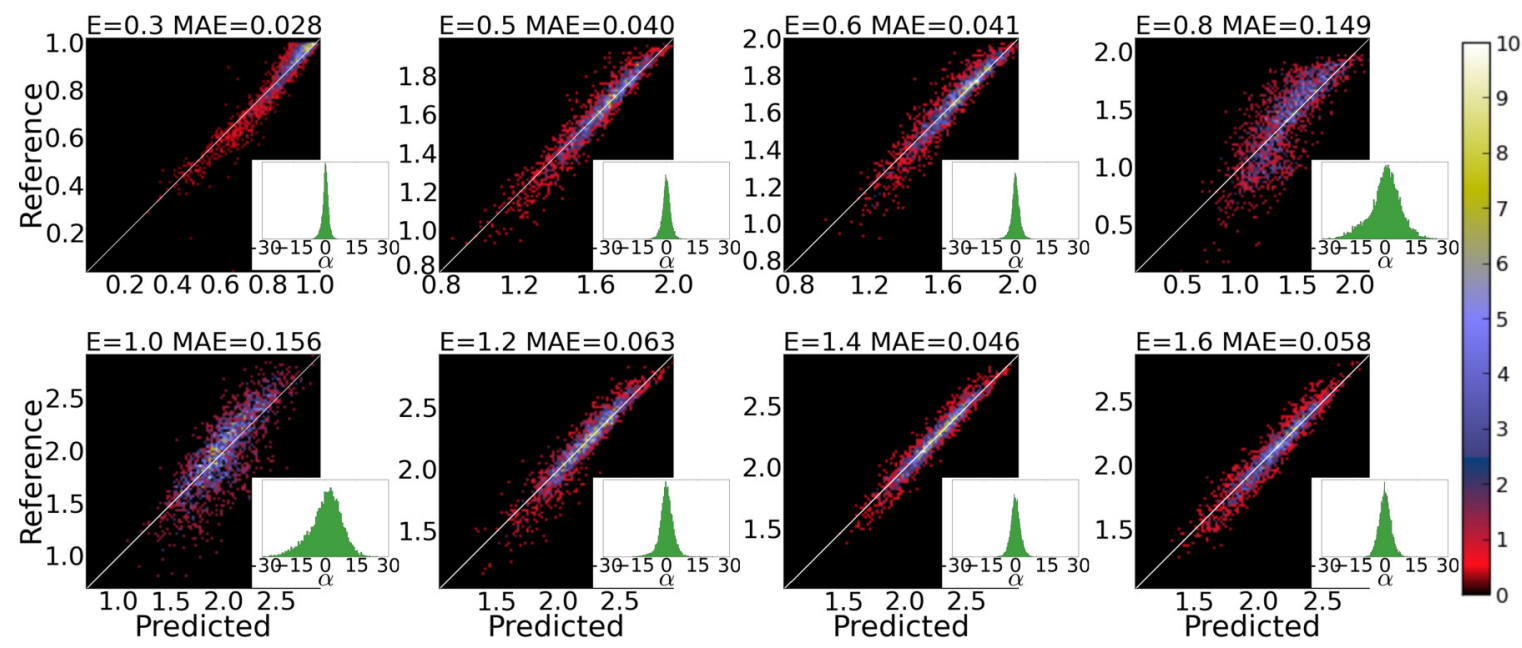

FIG. 2. (Color online) 2D histograms of correlated 2000 reference transmissions with machine-learning-based predicted values, for $N_{t}=$ 8000 samples with five spatially fixed scattering centers and parameters $\epsilon_{i}=0$ and $\gamma_{i j} \in[-1.5,-0.5]$. Above each panel the energy $E$ at which the transmissions were computed, and the mean absolute error (MAE), are indicated. Insets show the histograms of the corresponding $\left\{\alpha_{I}(E)\right\}$ distributions.

\section{A. First experiment}

We have obtained ML results for a training set consisting of $N_{t}=8000$ different samples, all with the same five impurity sites chosen at random, as also illustrated in Fig. 1(a). Hopping terms of the impurities to their nearest neighbors have been set randomly within the range $\gamma_{i j} \in[-1.5,-0.5]$. For the rest of sites in the leads and the channel, $\gamma_{i j}=-1$. On-site energies have been set to $\epsilon_{i}=0$ for all sites. The black line in Fig. 1(b) illustrates the stepwise increasing transmission of a defectless channel for increasing values of $E$ in the conduction band. Green lines show for defective channels the transmission drop as result of the backscattering, and represent the extent of the transmission variation for the considered range of energy. For various discrete values of $E$, highlighted with vertical blue lines in Fig. 1(b), we have trained a ML model and tested its performance for 2000 out-of-sample defected channels. Figure 2 shows the predictive performance in various panels, each corresponding to a different energy value, in heat histogram representation. Overall, the correlations between the ML (predicted) and Eq. (4) (reference) results are remarkable, with errors routinely scoring at less than $5 \%$ of the average transmission coefficients. Notice that the accuracy of the model varies as a function of electron energy, being the largest error at $E=0.8$ and $1 \mathrm{eV}$. This is also manifested by the corresponding $\left\{\alpha_{I}(E)\right\}$ histograms (insets of Fig. 2), which evince a direct relation between the broadening of the computed coefficients of Eq. (6) and the quality of the prediction. It is observed that $\alpha$ varies smoothly as a function of $E$, pointing out that the contribution of a given sample does not change abruptly as $E$ is tuned. This might help to improve the model's accuracy in the future through inclusion of derivatives of $\alpha$ with respect to $E$.

The two peaks in MAE in Fig. 3(b) are clearly consistent with the apparition of new transmission modes, which occur in the vicinity of $E=0.4$ and $0.8 \mathrm{eV}$. The wide variability of the transmission coefficients from sample to sample at a resonance energy and in the vicinity of a van Hove singularity hinder the training process and, thereby, enhance the unpredictability.
This can be ascribed to the interference phenomena resulting from the multiple reflections of electron waves with the scattering centers, rendering $T(E)$ strongly sensitive to both the distance between impurities and the strength of $\gamma_{i j}$. Notice the linear decrease of the MAE with logarithm of training set size for each $E$ value, including those of more difficult predictability.

\section{B. Second experiment}

Within the second experiment, we generated more complex training and test sets introducing an additional source of disorder. The scattering efficiency is enhanced by allowing the $\epsilon_{i}$ of the impurities to take finite values. Figure 1(c) features

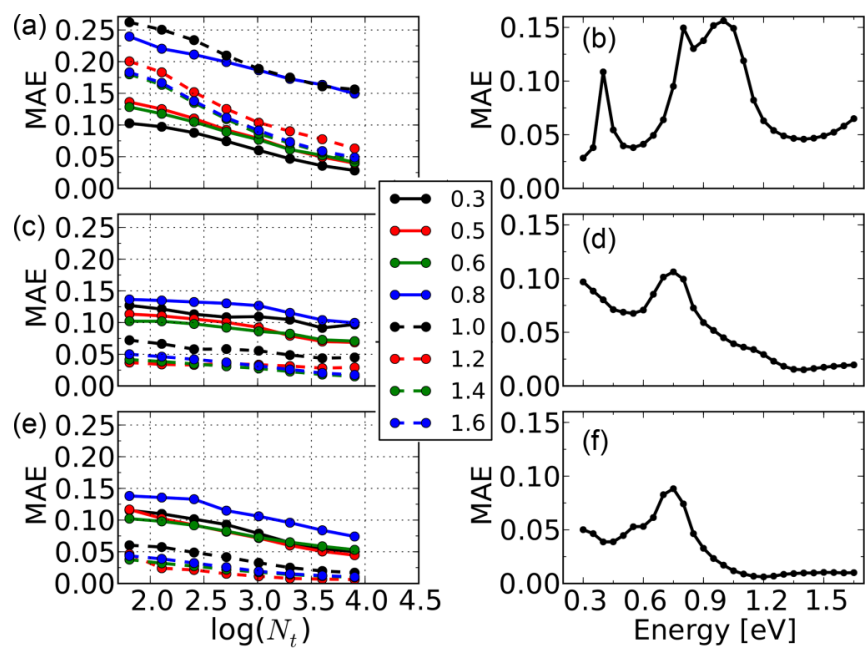

FIG. 3. (Color online) Linear drop of the mean absolute error (MAE) as a function of the logarithm of the number of training samples $\left(N_{t}\right)$, in (a) for $\epsilon_{i}=0$ and $\gamma_{i j} \in[-1.5,-0.5]$, in (c) for $\epsilon_{i} \in$ $[-13,0]$ and $\gamma_{i j} \in[-1,-0.3]$, and in (e) with the same parameters as in the latter after removal of $10 \%$ of the outliers. (b), (d), and (f) show the MAE as a function of the energy for $N_{t}=8000$ samples. 
the corresponding transmission profiles for $\epsilon_{i} \in[-13,0]$ and $\gamma_{i j} \in[-1,-0.3]$ for another training set with $N_{t}=8000$ samples. Figure 3(c) illustrates again a linear drop of the MAE with training set size. Despite the additional disorder, the higher degree of localization induced by the activation of the on-site energies leads to a significantly reduced MAE. For larger $N_{t}$, however, both models converge to similar error ranges. The $E$ dependency of the MAE is moderated in this case, exhibiting only a small peak at $E=0.8 \mathrm{eV}$, in the close vicinity to a new transmitting mode onset. Because small sample-to-sample variations may yield large differences in the transmission, some training data may be difficult to classify. This usually involves additional effort for the learning task, either in debasing the performance, or in slowing down the error decreasing with the training set size. A reduction of the variation range through the removal of $10 \%$ of outliers whose $T(E)$ deviate most from the average supports this surmise. Figures 3(e) and 3(f) show a significant improvement of the predictive power with reductions in the MAE by up to $50 \%$.

\section{Third experiment}

Finally, we investigate the ML model resulting from a training set with variable random positions of the scattering centers in combination with random $\gamma_{i j}$ strength. Specifically, we generated 100 samples with different random positions. From each of these samples we generated 100 defected configurations with $\gamma_{i j} \in[-1,-0.7]$ randomly chosen for the impurity sites, and $\gamma_{i j}=-1 \mathrm{eV}$ for all other sites. $\epsilon_{i}$ has been set to 0 throughout. Eight thousand out of the resulting 10000 channels have been selected at random to train our ML model, and the remaining 2000 were used for out-of-sample testing. The green lines of Fig. 4(a) feature the resulting transmission coefficients for this training set. The resulting evolution plot
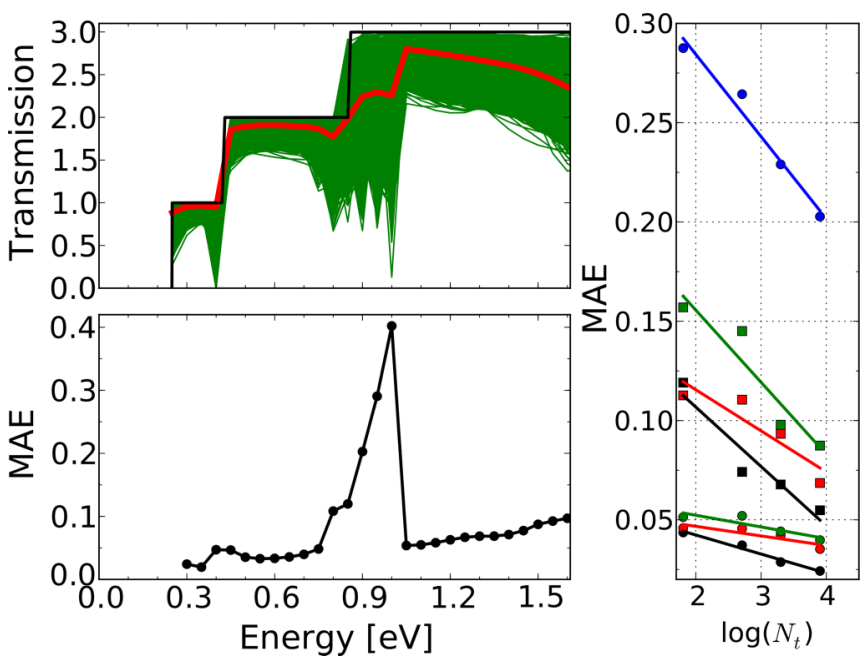

FIG. 4. (Color online) (a) Transmissions of $N_{t}=8000$ defective channels (green lines) with various $\gamma_{i j}$ strength and changeable random positions. Red line indicates the average. (b) shows the MAE as a function of the energy for the $N_{t}$ samples. (c) Linear drop of the mean absolute error (MAE) as a function of the logarithm of $N_{t}$ in (a). of the MAE with $N_{t}$ in Fig. 4(b) demonstrates a very good correlation with the predicted results. Only in the proximity of the resonance at $E=1 \mathrm{eV}$, however, the variation in $T$ becomes large, the MAE increasing by an order of magnitude to $\sim 0.4$. Despite such a large error, Fig. 4(c) clearly suggests that even at the resonance energy a lower error can be achieved through extension of the training set size.

Overall we have found that the error of the largest outlier in this study does not exceed $50 \%$ of the corresponding reference value. While such an error could be considered problematic for a deductive, physics-based, model, it is less surprising for a mere summation over weighted exponentials [Eq. (6)]. In terms of usefulness, such large outliers can still be considered acceptable in the context of virtual high-throughput screening [1], which is aimed at producing ranked lists of promising materials candidates for further in-depth study or experimental characterization. Furthermore, the implementation of more sophisticated ML techniques, currently used in active learning [6], such as calculating the predicted variance along with any transmission probability estimate, could be used in the future to assign error bars to out-of-sample predictions, and to remedy the issue through interactive and dynamic evolution of training set composition.

\section{CONCLUSION}

We have introduced a ML model for predicting electronic quantum transmission coefficients as a function of electron energy for one-dimensional channels. Our numerical results suggest that the model is capable of integrating previously computed transmission coefficient data into a simple and efficient framework, and of inferring transmission coefficients for new (out-of-sample) channels. The proposed descriptor has proved to be highly efficient in encoding the defected channels' identity. The remarkable performance of this ML scheme when it comes to capturing the complexity of interference phenomena lends further support to its viability in dealing with transport problems of undulatory nature. Furthermore, as follows from the different complexity of the equations to solve, the ML model is dramatically less computationally demanding than conventional models and, given a sufficiently large training set of disordered channels, yields competitive accuracy [17]. In summary, we have shown that nonlinear statistical regression approaches offer promising alternatives for solving the electron transmission problem in disordered nanostructures.

\section{ACKNOWLEDGMENTS}

Both authors acknowledge DOE BES glue funding through Grant No. FWP\#70081. This research used resources of the Argonne Leadership Computing Facility at Argonne National Laboratory, which is supported by the Office of Science of the U.S. DOE under Contract No. DE-AC02-06CH11357. O.A.vL. also acknowledges funding from the Swiss National Science foundation (No. PPOOP2_138932). 
[1] S. Curtarolo, G. L. W. Hart, M. B. Nardelli, N. Mingo, S. Sanvito, and O. Levy, Nature Mater. 12, 191 (2013).

[2] S. P. Ong, A. Jain, G. Hautier, M. Kocher, S. Cholia, D. Gunter, D. Bailey, D. Skinner, K. A. Persson, and G. Ceder, The Materials Project Appl. Phys. Lett. Mat. 1, 011002 (2011).

[3] Nature Mater. 12, 173 (2013).

[4] G. Hautier, C. C. Fischer, A. Jain, T. Mueller, and G. Ceder, Chem. Mater. 22, 3762 (2010).

[5] G. Pilania, C. Wang, X. Jiang, S. Rajasekaran, and R. Ramprasad, Sci. Rep. 3, 2810 (2013).

[6] T. Hastie, R. Tibshirani, and J. Friedman, The Elements of Statistical Learning: data mining, inference and prediction, Springer Series in Statistics (Springer, New York, 2001).

[7] K.-R. Müller, S. Mika, G. Rätsch, K. Tsuda, and B. Schölkopf, IEEE Transactions on Neural Networks 12, 181 (2001).

[8] M. Rupp, A. Tkatchenko, K.-R. Müller, and O. A. von Lilienfeld, Phys. Rev. Lett. 108, 058301 (2012).
[9] G. Montavon, M. Rupp, V. Gobre, A. Vazquez-Mayagoitia, K. Hansen, A. Tkatchenko, K.-R. Müller, and O. A. von Lilienfeld, New J. Phys. 15, 095003 (2013).

[10] J. C. Snyder, M. Rupp, K. Hansen, K.-R. Müller, and K. Burke, Phys. Rev. Lett. 108, 253002 (2012).

[11] R. Landauer, IBM J. Res. Dev. 1, 223 (1957).

[12] R. Landauer, Philos. Mag. 21, 863 (1970).

[13] M. Büttiker, Phys. Rev. Lett. 57, 1761 (1986).

[14] D. S. Fisher and P. A. Lee, Phys. Rev. B 23, 6851 (1981).

[15] O. A. von Lilienfeld, M. Rupp, and A. Knoll, arXiv:1307.2918 (2013).

[16] K. Hansen, G. Montavon, F. Biegler, S. Fazli, M. Rupp, M. Scheffler, O. A. von Lilienfeld, A. Tkatchenko, and K.-R. Müller, J. Chem. Theory Comput. 9, 3404 (2013).

[17] See Supplemental Material at http://link.aps.org/supplemental/ 10.1103/PhysRevB.89.235411 for a benchmark analysis of Green's functions vs ML approach performance. 